\section{Infections among Contacts of Patients with Nipah Virus, India}

\author{
Chong Tin Tan, Kum Thong Wong \\ Author affiliation: University of Malaya, Kuala Lumpur, Malaysia \\ DOI: https://doi.org/10.3201/eid2608.190722
}

To the Editor: Kumar et al. recently reported 3 asymptomatic, seropositive persons with Nipah virus (NiV) among 279 contacts of $18 \mathrm{NiV}$-infected patients (1). In the 1998-1999 NiV outbreak in Malaysia, asymptomatic, seropositive persons, most of whom were farm workers and soldiers involved in pig culling, also were identified $(2,3)$. Furthermore, some additional cases might not have been detected among patients who had mild, nonencephalitic symptoms (e.g., fever, influenza-like illness). Up to $16 \%$ of asymptomatic, seropositive persons exhibited lesions in their brain magnetic resonance imaging (MRI) scans, albeit a slightly smaller number than in patients with acute NiV encephalitis (2). These discrete, small highsignal lesions in the cerebrum were best seen on fluidattenuated inversion recovery images (2). In another study, a seropositive nurse who was exposed only to patients also had similar brain lesions visible on an MRI scan, suggesting person-to-person transmission (4). Whether late-onset NiV encephalitis (i.e., encephalitis in an asymptomatic or mildly symptomatic person) would develop in any of these persons, especially those with brain lesions visible on MRI scan, is unknown. In another cohort, approximately 5\% of asymptomatic or mildly symptomatic persons (5) had febrile encephalitis characterized by headache, seizures, focal neurologic signs, and cerebrospinal fluid pleocytosis, a set of symptoms and signs distinct from acute $\mathrm{NiV}$ encephalitis. In patients who survived acute NiV encephalitis, a clinicopathologically similar relapsing encephalitis has also been reported (5). The death rate was $\approx 20 \%$ during the clinical course of late-onset/relapsing $\mathrm{NiV}$ encephalitis (5).

Physicians should consider using brain MRI to identify subclinical brain lesions in asymptomatic, seropositive Nipah patients. Moreover, physicians should advise patients of the possibility that late-onset encephalitis might occur months or even years after virus exposure (5). New treatments are in development or on trial, so patients with these complications may be offered access to effective treatments.

\section{References}

1. Kumar CPG, Sugunan AP, Yadav P, Kurup KK, Aarathee R, Manickam P, et al. Infections among contacts of patients with Nipah virus, India. Emerg Infect Dis. 2019;25:1007-10. https://doi.org/10.3201/eid2505.181352

2. Tan KS, Ahmad Sarji S, Tan CT, Abdullah BJ, Chong HT, Thayapran T, et al. Patients with asymptomatic Nipah virus infection may have abnormal cerebral MR imaging. Neurology Journal of Southeast Asia. 2000;5:69-73.

3. Ali R, Mounts AW, Parashar UD, Sahani M, Lye MS, Isa MM, et al. Nipah virus among military personnel involved in pig culling during an outbreak of encephalitis in Malaysia, 1998-1999. Emerg Infect Dis. 2001;7:759-61. https://doi.org/10.3201/eid0704.017433

4. Tan CT, Tan KS. Nosocomial transmissibility of Nipah virus. J Infect Dis. 2001;184:1367. https:/ / doi.org/10.1086/323996

5. Chong HT, Tan CT. Relapsed and late-onset Nipah encephalitis, a report of 3 cases. Neurology Journal of Southeast Asia. 2003;8:109-12.

Address for correspondence: Kum Thong Wong, Department of Pathology, Faculty of Medicine, University of Malaya, 50603 Kuala Lumpur, Malaysia; email: wongkt@um.edu.my 